\title{
Outbreak due to a Klebsiella pneumoniae strain harbouring KPC-2 and VIM-1 in a German university hospital, July 2010 to January 2011
}

J Steinmann (joerg.steinmann@uk-essen.de) ${ }^{1}$, M Kaase $^{2}$, S Gatermann², W Popp ${ }^{3}$, E Steinmann ${ }^{4}$, M Damman ${ }^{5}$, A Paul5, F Saner ${ }^{5}$, J Buer ${ }^{1}$, P M Rath ${ }^{1}$

1. Institute of Medical Microbiology, University Hospital Essen, University of Duisburg- Essen, Essen, Germany

2. National Reference Laboratory for Multidrug Resistant Gram negative Bacteria, Department of Medical Microbiology, Ruhr University Bochum, Bochum, Germany

3. Hospital Hygiene, University Hospital Essen, University of Duisburg-Essen, Essen, Germany

4. Twincore Center for Experimental and Clinical Infection Research, Hannover, Germany

5. Department of General, Visceral and Transplant Surgery, University Hospital Essen, University of Duisburg-Essen, Essen, Germany

Citation style for this article:

Steinmann J, Kaase M, Gatermann S, Popp W, Steinmann E, Damman M, Paul A, Saner F, Buer J, Rath PM. Outbreak due to a Klebsiella pneumoniae strain harbouring KPC-2 and VIM-1 in a German university hospital, July 2010 to January 2011.

EEuro Surveill. 2011;16(33):pii=19944. Available online: http://www.eurosurveillance.org/ViewArticle.aspx?Articleld=19944

Article published on 18 August 2011

We describe the epidemiology and characteristics of the pathogen and patients $(n=7)$ associated with an outbreak of a carbapenem-resistant Klebsiella pneumoniae (CRKP) strain in a German university hospital from July 2010 to January 2011. Species identification and detection of carbapenem resistance were carried out using standard microbiological procedures. Carbapenemases were detected by phenotypic methods and specific polymerase chain reactions (PCRs). DNA fingerprinting profiles were performed with repetitive sequence-based PCR. Medical records of colonised or infected patients were retrospectively reviewed. Antibiotic resistance profiles, PCR-specific amplification products and genotyping demonstrated that the outbreak occurred because of the spread of a single CRKP clone harbouring both KPC-2 and VIM1. Five of the seven patients had invasive infections with the CRKP strain; the deaths of four of them were directly related to the infection. Early implementation of infection control interventions brought about efficient containment of further cross-transmission. Rapid dissemination of carbapenemase-producing Enterobacteriaceae is a serious concern in patient care and is a problem that has emerged in western Europe.

\section{Introduction}

Enterobacteriaceae are widespread worldwide and frequently exhibit resistance to extended-spectrum beta-lactamases (ESBLs) [1]. The first-line treatments for infections caused by ESBL-producing strains are carbapenems. The frequent use of carbapenems, however, combined with the transmissibility of resistance determinants mediated by plasmids, transposons and gene cassettes, has contributed to the increase of carbapenem resistance by Enterobacteriaceae [2,3]. In countries such as the United States, Israel and Greece, carbapenemase-producing Enterobacteriaceae have emerged and the dissemination of these multidrugresistant pathogens has become a problem in the clinical care of patients and in public health [4-6].

Invasive infections with these organisms have been associated with high rates of morbidity and mortality, due to their resistance to most available antimicrobial agents [7-9]. Patients who are hospitalised for prolonged periods and those with severe underlying disease are at high risk of acquiring these types of pathogens [10].

The Ambler classification describes various classes of carbapenemases [11]. Klebsiella pneumoniae carbapenemases (KPC) belong to class A; they are increasingly prevalent in parts of the United States, Israel and Greece and are also an emerging concern in western Europe [12]. Class B metallo-beta-lactamases of the types IMP (imipenemase) and VIM (Verona integronencoded metallo-beta-lactamase) are common in non-fermentative bacteria and have been recently recognised in Enterobacteriaceae worldwide [12]. Since 2003, Enterobacteriaceae-producing metallo-betalactamases of the VIM type have begun to spread in Greek hospitals [4]. During the first few months of 2010, K. pneumoniae strains carrying both VIM and KPC were identified in Greek hospitals [12]. Strains carrying the recently reported New Delhi metallobeta-lactamase 1 (NDM-1), found throughout India, Pakistan and Bangladesh, have also been rapidly disseminated to Europe and across the globe [13]. OXAtype (oxacillin-hydrolysing) carbapenemases (Class D) are widespread and have been detected primarily in Enterobacteriaceae and in Pseudomonas aeruginosa [14]. The Ambler classification also describes Class C (AmpC-type beta-lactamases), but these beta-lactamases can only hydrolyse broad- and extended-spectrum cephalosporins, not carbapenems. 
Carbapenem-non-susceptible Enterobacteriaceae have been sporadically reported in Germany in recent years. In 2008, an outbreak with nine patients affected by a $K$. pneumoniae strain producing a KPC-2 occurred in the southern part of Germany [15]. In 2009 and 2010, two outbreaks in eastern Germany with a K. pneumoniae producing KPC-3 were reported, where in 2009 more than 10 patients were affected and in 2010 less than 10 patients (personal communication, Y. Pfeifer, 18 July 2011).

Here we report on an outbreak of a multidrug-resistant $K$. pneumoniae strain harbouring KPC-2 and VIM1 , which affected nine patients in the intensive care unit (ICU) of the Department of General, Visceral and Transplant Surgery in a university hospital in western Germany from July 2010 to January 2011. To the best of our knowledge, this is the first report of the detection of a carbapenem-resistant Enterobacteriaceae producing both KPC-2 and VIM-1 outside of Greece.

\section{Methods}

\section{Setting}

The University Hospital Essen is a German tertiarycare teaching hospital with 1,300 beds; the hospital treats approximately 50,000 inpatients per year. The Department of General, Visceral and Transplant Surgery in the University Hospital Essen is one of the leading liver transplant centres in Europe (158 liver transplants were performed in 2010) and it has a 10-bed ICU. Since January 2000, cultures of nasopharyngeal swabs from all patients on admission to the ICU have been screened once for meticillin-resistant Staphylococcus aureus. The natural habitat of Enterobacteriaceae is the human gastrointestinal tract and the most practical way to detect carbapenem-resistant Enterobactericeae is to analyse perianal or rectal swabs. Before the outbreak described in this report, no screening for surveillance of carbapenem-resistant Enterobacteriaceae was performed in the ICU.
Bacterial strain identification and susceptibility testing

All detected isolates were identified using standard conventional microbial methods (e.g. colony morphologies, pigment production, etc.) [16]. Identity confirmation and susceptibility testing were performed using the semi-automated systems VITEK 2 (bioMérieux, Germany) and MicroScan WalkAway (Siemens, Germany). Minimum inhibitory concentrations (MICs) of meropenem and imipenem were also determined using Etest (AB Biodisk, Sweden) on Mueller-Hinton agar.

Susceptibility results were interpreted using following clinical breakpoints of the European Committee on Antimicrobial Susceptibility Testing (EUCAST): $\leq 2 \mathrm{mg} / \mathrm{L}$ for meropenem-susceptibility, $\geq 8 \mathrm{mg} / \mathrm{L}$ for meropenemresistance, $\leq 2 \mathrm{mg} / \mathrm{L}$ for imipenem-susceptibilty and $\geq 8$ $\mathrm{mg} / \mathrm{L}$ for imipenem-resistance [17].

\section{Detection of carbapenemases and metallo-beta-lactamases}

The modified Hodge test was performed as previously described [18]. Additionally, a combined disk test for imipenem and meropenem was performed with $930 \mu \mathrm{g}$ ethylene diamine tetraacetic acid (EDTA) and $300 \mu \mathrm{g}$ 3 -aminophenylboronic acid $[19,20]$. An increase of the zone inhibition diameter $\geq 7 \mathrm{~mm}$ for EDTA and $\geq 4 \mathrm{~mm}$ for 3-aminophenylboronic acid was considered positive.

PCRs were performed for bla $a_{\mathrm{KPC}}, b l a_{\mathrm{VIM}}, b l a_{\mathrm{IMP}}$ and bla $a_{\mathrm{OXA}}-48$ genes and amplicons were sequenced as previously described [19,21-23].

\section{Genotyping}

Genotyping of all detected isolates was performed by repetitive sequence-based PCR using the semi-automated system DiversiLab (bioMérieux) and the bacterial DNA was amplified with the DiversiLab Klebsiella kit. The amplified fragments were separated and fluorescence intensities were detected with a microfluidic chip and a B 2100 bioanalyzer (Agilent Technologies,

\section{TABLE}

Characteristics of patients with carbapenem-resistant Klebsiella pneumoniae, university hospital, Germany, July 2010January $2011(n=7)$

\begin{tabular}{|c|c|c|c|c|c|}
\hline $\begin{array}{l}\text { Patient } \\
\text { number }\end{array}$ & Underlying condition & Initial specimen & Infection/colonisation & Antibiotic therapy & Status on hospital discharge \\
\hline 1 & $\begin{array}{c}\text { Solid organ } \\
\text { transplantation }\end{array}$ & Blood & Bloodstream infection & Tigecycline,colistin & Dead - death unrelated to CRKP \\
\hline 2 & Cancer & Pleural fluid & Pleural infection & Fosfomycin & Dead - death related to CRKP \\
\hline 3 & $\begin{array}{c}\text { Solid organ } \\
\text { transplantation }\end{array}$ & Blood & Bloodstream infection & Tigecycline,colistin & Dead - death related to CRKP \\
\hline 4 & $\begin{array}{c}\text { Solid organ } \\
\text { transplantation }\end{array}$ & Blood & Bloodstream infection & Colistin,amikacin & Dead - death related to CRKP \\
\hline 5 & $\begin{array}{l}\text { Solid organ } \\
\text { transplantation }\end{array}$ & Rectal & Colonisation & None & Alive \\
\hline 6 & Cancer & Rectal & Colonisation & None & Alive \\
\hline 7 & $\begin{array}{c}\text { Solid organ } \\
\text { transplantation }\end{array}$ & $\begin{array}{l}\text { Tracheal } \\
\text { secretion }\end{array}$ & Bloodstream infection & $\begin{array}{l}\text { Tigecycline, colistin, } \\
\text { amikacin }\end{array}$ & Dead - death related to CRKP \\
\hline
\end{tabular}


United States). The results were illustrated with the web-based DiversiLab software version v.r.3.3.40.

The Pearson correlation coefficient and the unweightedpair group method with arithmetic mean were used to determine the genetic similarity of the tested samples. A clinical carbapenem-susceptible $K$. pneumoniae strain served as a control, to underpin the discriminatory power of the method. A similarity cut-off of $95 \%$ was considered indicative of clonal relatedness, as described previously $[24,25]$.

\section{Results}

Outbreak description and

epidemiological investigations

Carbapenem-resistant $K$. pneumoniae (CRKP) strains were isolated from seven patients hospitalised in the ICU of the Department of General, Visceral and Transplant Surgery of the University Hospital Essen. The age of these patients (five men and two women) ranged from 22 to 72 years. The patient's characteristics are shown in the Table. Bloodstream infections with the CRKP strain occurred in two kidney transplant recipients and two liver transplant recipients. The deaths of three of the four patients were directly related to their CRKP infections.

The first patient (Patient 1) from whom the CRKP strain was isolated (from blood) had received a solid organ transplant in June 2010 and experienced fever and lung infiltrates during the postoperative course. This patient was originally from Germany and there was no evidence that they had travelled to southern Europe, the United States or east Asia in recent years. Nearly two weeks after this first isolation of the CRKP strain was identified, two patients in the ICU (Patients 2 and 3) were found to have invasive infections with this strain,

\section{FIGURE 1}

Synoptic curve of patients with carbapenem-resistant Klebsiella pneumoniae, university hospital, Germany, July 2010-January $2011(n=7)$

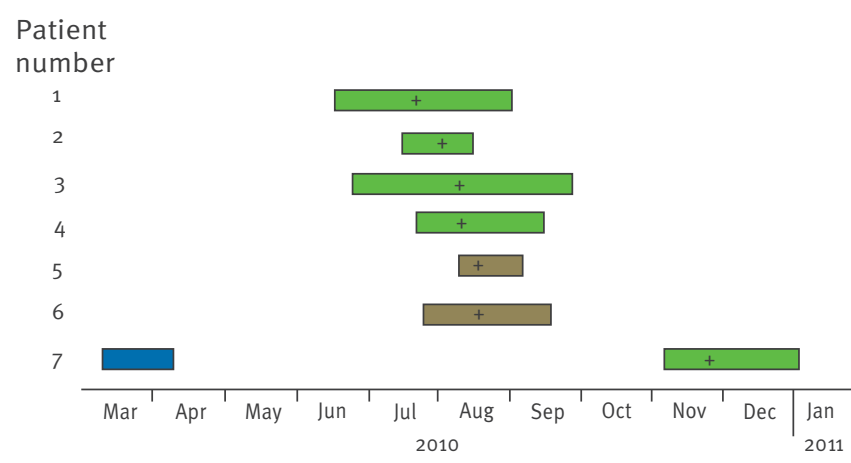

Date of first CRKP-positive specimen

\footnotetext{
Period of first stay in the hospital

$\square$ Patients with invasive CRKP infection

$\square$ Patients with CRKP colonisation

+ Date specimen tested positive for CRKP
}

although they were not in the same ICU room as the first affected patient. During the subsequent 12 days, three additional patients (Patients 4-7) tested positive for the CRKP strain, two of whom were colonised only rectally. The synoptic curve of all seven patients is shown in Figure 1.

A patient from Greece, who had been previously hospitalised in Greece (Patient 7), received a solid organ transplant in March 2010, three months before the Klebsiella outbreak occurred. At that time, this patient had Enterobacter cloacae bacteraemia, but during this period, no CRKP strain was isolated from this patient or from other ICU inpatients. At the end of March, the patient was discharged, in a good medical condition. In November 2010, this person was again hospitalised at the University Hospital Essen. The patient had travelled to Greece between the two hospitalisations in Germany and had had contact with the healthcare system in Greece during the interval between hospitalisation. The patient also developed bacteraemia with the CRKP strain and died at the beginning of January 2011.

Antimicrobial identification and susceptibility All studied isolates were identified as K. pneumoniae. They exhibited the same multidrug-resistant profile, which showed resistance to all beta-lactams, including imipenem and meropenem ( $\mathrm{MIC} \geq 8 \mathrm{mg} / \mathrm{L}$ ), in the semi-automated systems. The Etest yielded MIC values of $\geq 32 \mathrm{mg} / \mathrm{L}$ for imipenem and meropenem. All CRKP isolates, with the exception of one isolate from Patient 7, were found to be susceptible to colistin, tigecycline and fosfomycin according to the EUCAST breakpoints. Susceptibility testing of this isolate from this patient demonstrated resistance to colistin (MIC $>2 \mathrm{mg} / \mathrm{L}$ ) and fosfomycin (MIC >32 mg/L).

\section{Carbapenemase characterisation}

All isolates were screened for carbapenemase production using phenotypic and molecular methods. The modified Hodge test yielded positive results for all isolates, indicating the presence of a carbapenemase. However, the combined disk test with EDTA for the detection of metallo-beta-lactamases was positive only in one isolate and only for meropenem. The combined disk test with 3-aminophenylboronic acid for the detection of KPC enzymes was negative for all isolates. By PCR and subsequent sequencing, bla $a_{\mathrm{KPC}}{ }^{-2}$ and $b / a_{\mathrm{VIM}}{ }^{-1}$ genes were found in all isolates.

\section{DNA fingerprinting}

As depicted in Figure 2, semi-automated repetitive sequence-based PCR demonstrated that all VIM-1producing and KPC-2-producing $K$. pneumoniae isolates had indistinguishable band patterns, with a percentage similarity higher than $95 \%$, which indicates clonal relatedness. The CRKP strain from Patient 7 , which was the last isolate detected during the outbreak, was also clonally related to the other isolates.

CRKP: carbapenem-resistant Klebsiella pneumoniae. 
Infection control measures and interventions After the second and third CRKP isolates were detected in routinely collected samples, an outbreak control team was organised and infection control strategies were established and implemented (Box).

All CRKP-positive patients were isolated in single-bed rooms, where strict contact precautions were maintained. Barrier nursing (i.e. use of gowns, masks and gloves) was used and contact precautions were also implemented for patients who had shared a room with a CRKP-positive patient. The transfer of CRKP-positive patients to other units or hospitals was discontinued.

To identify the prevalence of CRKP colonisation/infection, samples for cultures from various body sites (nasopharynx, urinary tract and rectum) were collected once a week from all ICU inpatients and all patients who had been moved to a different ward in the hospital. A patient was considered to be CRKP negative if all cultures of samples collected from the three body sites were found to be negative on three different days with a two-day interval between sampling. All surveillance samples were cultured on an internally validated selective medium containing imipenem, vancomycin and amphotericin B for specific detection of carbapenemresistant Gram-negative bacterial rods. Furthermore, environmental sites were investigated for CRKP contamination. In total, 32 patients and 68 environmental sites were screened during the outbreak period. The environmental samples were taken from medical devices (e.g. X-ray apparatus and syringe pumps), personal computers, telephones, door handles, floors and various other surfaces in the bathrooms or offices in the ICU. Screening of cultures from samples collected from all inpatients in the ICU detected two

\section{FIGURE 2}

DNA fingerprinting of carbapenem-resistant Klebsiella pneumoniae isolates by repetitive sequence-based PCR, university hospital, Germany, July 2010-January 2011 (n=6)

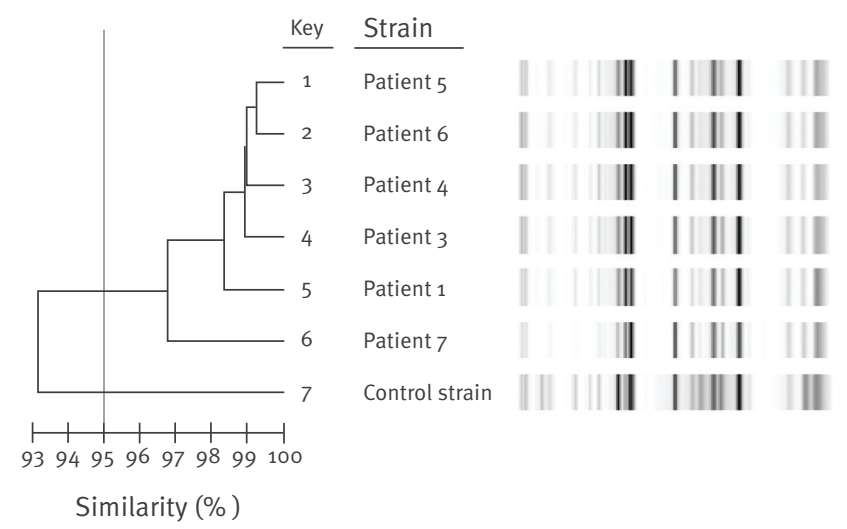

The dendrogram and gel image demonstrate strain clustering. The horizontal bar on the bottom left indicates the percentage similarity within the strains. A cut-off of $95 \%$ similarity (vertical line) was chosen for determination of clonal relatedness. A clinical carbapenem-susceptible $K$. pneumoniae strain served as a control. a One carbapenem-resistant Klebsiella pneumoniae isolate (from Patient 2) could not be saved for DNA fingerprinting. additional colonised patients (Patients 5 and 6) who had not previously been found to harbour CRKP and had not initially been treated with contact precautions. Environmental sampling demonstrated the presence of Acinetobacter baumannii and Staphylococcus aureus on medical equipment, but no carbapenem-resistant Enterobacteriaceae were detected.

All ICU personnel, including cleaning staff and physical therapists, were instructed about standard hygiene procedures such as appropriate hand hygiene. In addition, the number of nursing staff in the ICU was increased during the outbreak period so that the increased nursing effort for isolated patients could be managed.

\section{Discussion}

The epidemiological and molecular investigations showed that a single $K$. pneumoniae strain that produced both VIM-1 and KPC-2 was responsible for the outbreak. To the best of our knowledge, the occurrence of VIM-1-positive and KPC-2-positive $K$. pneumoniae isolates has previously been reported only in Greece $[26,27]$.

In July 2010, the first patient developed an infection with the VIM-1- and KPC-2-positive CRKP outbreak strain and cross-transmission occurred thereafter. In November 2010, Patient 7, who had been an inpatient in March 2010, was again hospitalised in the CRKPaffected ICU after being discharged from a Greek hospital. The patient also had bacteraemia caused by the CRKP outbreak strain. Our investigations yielded no proof that the CRKP strain originally came from another country; in our opinion, it might be possible that the strain could had been transferred to the German hospital in March 2010 during the first hospital stay of the patient and had remained undetected until July. As described in several previous reports, importation of carbapenem-resistant strains into other European

\section{Box}

Interventions implemented for control of an outbreak due to carbapenem-resistant Klebsiella pneumoniae in an intensive care unit, university hospital, Germany, July 2010-January 2011

- Isolating patients in single rooms or isolating cohorts with the same CRKP species and same resistance profile

- Obtaining targeted surveillance samples from all inpatients and contact patients

- Stopping the transfer of CRKP-positive patients to other units or hospitals

- Increasing the number of nursing staff in the intensive care unit during the outbreak period

- Instructing personnel, including cleaning staff and physical therapists, about standard hygiene procedures (e.g. appropriate hand hygiene)

- Disinfecting rooms with $3 \%$ nebulised Pentakaliumbis(peroxymonosulfate)-bis(sulfate) and cleaning rooms with $0.5 \%$ Pentakalium-bis(peroxymonosulfate)bis(sulfate) after discharge of a CRKP-positive patient

CRKP: carbapenem-resistant Klebsiella pneumoniae. 
countries by patients who had been previously hospitalised in Greece is not uncommon [15,28-32].

Due to the limited number of patients, we did not carry out a case-control study. We did notice, however, that recipients of solid organ transplants were particularly affected by the CRKP strain during the outbreak and developed invasive infections. As has been reported by others, such transplant recipients are frequently affected by carbapenem-resistant Enterobacteriaceae. For example, a study from a liver transplant centre in France found that, in 2003 and 2004 , eight patients were infected with an imipenem-resistant, VIM-1-producing $K$. pneumoniae isolate; seven of these patients had undergone liver transplantation [32]. The index patient in that outbreak was a liver transplant recipient, who had been transferred from Greece to France. In addition, in the first described German outbreak with a KPC2-positive $K$. pneumoniae, two of nine affected patients had received solid organ transplants [15].

Recipients of solid organ transplants are routinely and extensively given broad-spectrum anti-infective agents as prophylaxis or targeted therapy during the preoperative, perioperative and postoperative periods. Broadspectrum antibiotic therapy exerts a selective pressure towards resistant organisms and affects the normal body flora. Furthermore, transplantation, immunosuppressive therapy and intensive care medicine, including use of medical devices, allow pathogens to colonise or infect patients. Therefore, patients who have received solid organ transplants may be at higher risk of acquiring carbapenem-resistant Enterobacteriaceae in outbreak situations than are inpatients who have not received such transplants.

Laboratory identification of carbapenemase-producing Enterobacteriaceae is challenging. Several bacteria produce carbapenemases and there is a high degree of diversity of these enzymes. As shown in our study and reported by others, the negative results of EDTA or boronic acid-based testing of the CRKP isolates may be attributed to the masking effect of the co-produced carbapenemases $[26,27]$. These concerns must also be considered when carbapenemase-producing isolates are detected. Microbiology laboratories without the necessary expertise are urged to send potential strains to reference laboratories for appropriate testing. It should be noted that early detection of carbapenemresistant isolates is of utmost importance in allowing adequate antimicrobial therapy to be initiated and in avoiding cross-transmission.

Containment of the outbreak began after the establishment of an outbreak control team that initiated infection control interventions. It is well known that hospital staff are potential vectors for transmission of resistant organisms, with documented carriage on hands or clothing [33]. However, we decided not to screen hospital staff for hand carriage because no legal guidelines exist in Germany on how to deal with the affected persons. However, additional cross-transmission was then prevented by strict patient isolation, intensified hand disinfection and the routine analysis of targeted surveillance cultures.

This outbreak led us to screen immunosuppressed patients or organ transplant recipients in ICUs for carbapenem-resistant Enterobacteriaceae by taking rectal swabs once a week and to monitor the course of colonisation or infection. We strongly recommend the use of perianal surveillance cultures, especially for patients who have been previously hospitalised in countries in which carbapenemase producers are widespread (e.g. Greece, Israel and the United States). Preventive contact precautions should be carried out for such patients until the results of microbiology studies have been obtained. In France, for example, carbapenemase-producing Enterobacteriaceae emerged in 2004 and the French Institute for Public Health Surveillance (Institut de Veille Sanitaire, InVS), recently recommended reinforcement of screening and control measures at national level when cross-border transfer of patients occurs [34].

For the microbiological analysis of targeted surveillance cultures, we suggest using a selective medium containing imipenem, vancomycin and amphotericin B so that the growth of Gram-positive and carbapenemsusceptible Gram-negative bacteria and even fungi in rectal flora can be avoided. Other authors have reported that media containing cefotaxime or ceftazidime are also feasible for the detection of multidrugresistant Enterobacteriaceae [35].

In summary, a VIM-1- and KPC-2-positive $K$. pneumoniae strain was responsible for an outbreak that affected seven patients in one ICU.

Rapid dissemination of carbapenemase-producing Enterobacteriaceae is a serious concern in clinical patient care and these pathogens are now also present in western Europe. Therefore, using surveillance cultures and initiating strict hygiene procedures is mandatory for the prevention and early detection of carbapenem-resistant Enterobacteriaceae in units where high-risk patients receive care.

Acknowledgements

We thank D. Schmidt for excellent technical assistance.

References

1. Paterson DL, Bonomo RA. Extended-spectrum betalactamases: a clinical update. Clin Microbiol Rev. 2005;18(4):657-86.

2. Kang HY, Jeong YS, Oh JY, Tae SH, Choi CH, Monn DC, et al. Characterization of antimicrobial resistance and class 1 integrons found in Escherichia coli isolates from humans and animals in Korea. J Antimicrob Chemother. 2005;55(5):639-44

3. Schwaber MJ, Carmeli Y. Carbapenem-resistant Enterobacteriaceae: a potential threat. JAMA. 2008;300(24):2911-3. 
4. Vatopoulos A. High rates of metallo-beta-lactamaseproducing Klebsiella pneumoniae in Greece--a review of the current evidence. Euro Surveill. 2008;13(4):8023. Available from: http://www.eurosurveillance.org/ViewArticle. aspx?Articleld $=8023$

5. Kitchel B, Rasheed JK, Patel JB, Srinivasan A, Navon-Venezia S, Carmeli Y, et al. Molecular epidemiology of KPC-producing Klebsiella pneumoniae isolates in the United States: clonal expansion of multilocus sequence type 258. Antimicrob Agents Chemother. 2009;53(8):3365-70.

6. Leavitt A, Carmeli Y, Chmelnitsky I, Goren MG, Ofek I, Navon-Venezia S. Molecular epidemiology, sequence types, and plasmid analyses of KPC-producing Klebsiella pneumoniae strains in Israel. Antimicrob Agents Chemother 2010;54(7):3002-6.

7. Borer A, Saidel-Odes L, Riesenberg K, Eskira S, Peled N, Nativ $\mathrm{R}$, et al. Attributable mortality rate for carbapenem-resistant Klebsiella pneumoniae bacteremia. Infect Control Hosp Epidemiol. 2009;30(10):972-6.

8. Daikos GL, Petrikkos P, Psichogiou M, Kosmidis C, Vryonis $\mathrm{E}$, Skoutelis A, et al. Prospective observational study of the impact of VIM-1 metallo-beta-lactamase on the outcome of patients with Klebsiella pneumoniae bloodstream infections. Antimicrob Agents Chemother. 2009;53(5):1868-73.

9. Patel G, Huprikar S, Factor SH, Jenkins SG, Calfee DP. Outcomes of carbapenem-resistant Klebsiella pneumoniae infection and the impact of antimicrobial and adjunctive therapies. Infect Control Hosp Epidemiol. 2008;29(12):1099-106.

10. Souli M, Galani I, Antoniadou A, Papadomichelakis E, Poulakou G, Panagea T, et al. An outbreak of infection due to betalactamase Klebsiella pneumoniae carbapenemase 2-producing K. pneumoniae in a Greek University Hospital: molecular characterization, epidemiology, and outcomes. Clin Infect Dis. 2010;50(3):364-73.

11. Ambler RP. The structure of beta-lactamases. Philos Trans R Soc Lond B Biol Sci. 1980;289(1036):321-31.

12. Grundmann H, Livermore DM, Giske CG, Canton R, Rossolini GM, Campos J, et al. Carbapenem-non-susceptible Enterobacteriaceae in Europe: conclusions from a meeting of national experts. Euro Surveill. 2010;15(46):pii=19711. Available from: http://www.eurosurveillance.org/ViewArticle. aspx?Articleld $=19711$

13. Moellering RC Jr. NDM-1--a cause for worldwide concern. N Engl J Med. 2010;363(25):2377-9.

14. Naas T, Nordmann P. OXA-type beta-lactamases. Curr Pharm Des. 1999;5(11):865-79.

15. Wendt C, Schütt S, Dalpke AH, Konrad M, Mieth M, TrierweilerHauke $B$, et al. First outbreak of Klebsiella pneumoniae carbapenemase (KPC)-producing K. pneumoniae in Germany. Eur J Clin Microbiol Infect Dis. 2010;29(5):563-70.

16. Murray PR, Baron EJ, Jorgensen JH, Landry ML, Pfaller MA. Manual of clinical microbiology. 9th ed, Herndon: ASM Press, 2007.

17. European Committee on Antimicrobial Susceptibility Testing (EUCAST). Clinical breakpoints. EUCAST. [Accessed 20 Jan 2011]. Available from: http://www.eucast.org/ clinical_breakpoints

18. Clinical Laboratory Standards Institute (CLSI). Performance standards for antimicrobial susceptibility testing: nineteenth informational supplement. CLSI document M100-S19. Wayne: CLSI; 2009. p. 136-9.

19. Pitout JD, Gregson DB, Poirel L, McClure JA, Le P, Church DL. Detection of Pseudomonas aeruginosa producing metallo-betalactamases in a large centralized laboratory. J Clin Microbiol. 2005;43(7):3129-35.

20. Pasteran F, Mendez T, Guerriero L, Rapoport M, Corso A. Sensitive screening tests for suspected class A carbapenemase production in species of Enterobacteriaceae. J Clin Microbiol. 2009.;47(6):1631-9.

21. Yigit H, Queenan AM, Anderson GJ, Domenech-Sanchez A, Biddle JW, Steward CD, et al. Novel carbapenem-hydrolyzing beta-lactamase, KPC-1, from a carbapenem-resistant strain of Klebsiella pneumoniae. Antimicrob Agents Chemother. 2001;45(4):1151-61.

22. Juan C, Beceiro A, Gutiérrez O, Albertí S, Garau M, Pérez JL, et al. Characterization of the new metallo-beta-lactamase VIM-13 and its integron-borne gene from a Pseudomonas aeruginosa clinical isolate in Spain. Antimicrob Agents Chemother. 2008;52(10):3589-96.

23. Poirel L, Héritier C, Tolün V, Nordmann P. Emergence of oxacillinase-mediated resistance to imipenem in Klebsiella pneumoniae. Antimicrob Agents Chemother. 2004;48(1):15-22.
24. Tenover FC, Gay EA, Frye S, Eells SJ, Healy M, McGowan JE Jr. Comparison of typing results obtained for methicillinresistant Staphylococcus aureus isolates with the DiversiLab system and pulsed-field gel electrophoresis. J Clin Microbiol. 2009;47(8):2452-7.

25. Marchaim D, Chopra T, Pogue JM, Perez F, Hujer AM, Rudin S, et al. Outbreak of colistin-resistant, carbapenem-resistent Klebsiella pneumoniae in metropolitan Detroit, Michigan. Antimicrob Agents Chemother. 2011;55(2):593-9.

26. Giakkoupi P, Pappa O, Polemis M, Vatopoulos AC, Miriagou $\mathrm{V}$, Zioga A, et al. Emerging Klebsiella pneumoniae isolates coproducing KPC-2 and VIM-1 carbapenemases. Antimicrob Agents Chemother. 2009;53(9):4048-50.

27. Zioga A, Miriagou V, Tzelepi E, Douzinas E, Tsakiri M, Legakis NJ, et al. The ongoing challenge of acquired carbapenemases: a hospital outbreak of Klebsiella pneumoniae simultaneously producing VIM-1 and KPC-2. Int J Antimicrob Agents. 2010;36(2):190-1.

28. Österblad M, Kirveskari J, Koskela S, Tissari P, Vuorenoja K, Hakanen AJ, et al. First isolations of KPC-2-carrying ST258 Klebsiella pneumoniae strains in Finland, June and August 2009. Euro Surveill. 2009;14(40):pii=19349. Available from: http://www.eurosurveillance.org/ViewArticle. aspx?Articleld $=19349$

29. Samuelsen $\varnothing$, Naseer U, Tofteland S, Skutlaberg DA, Onken A, Hjetland R, et al. Emergence of clonally related Klebsiella pneumoniae isolates of sequence type 258 producing plasmidmediated KPC carbapenemase in Norway and Sweden. J Antimicrob Chemother. 2009;63(4):654-8.

30. Hasman H, Fjeldsøe-Nielsen H, Lausten T, Berthelsen BG. Isolation of a VIM-1 metallo- $\beta$-lactamse-producing Klebsiella pneumoniae isolate in Denmark. Int J Antimicrob Agents. 2010;36(5):468-9.

31. Barbier F, Ruppé E, Giakkoupi P, Wildenberg L, Lucet J, Vatopoulos A, et al. Genesis of a KPC-producing Klebsiella pneumoniae after in vivo transfer from an imported Greek strain. Euro Surveill. 2010;15(1):pii=19457. Available from: http://www.eurosurveillance.org/ViewArticle. aspx?Articleld $=19457$

32. Kassis-Chikhani N, Saliba F, Carbonne A, Neuville S, Decre $D$, Sengelin C, et al. Extended measures for controlling an outbreak of VIM-1 producing imipenem-resistant Klebsiella pneumoniae in a liver transplant centre in France, 2003-2004. Euro Surveill. 2010;15(46):pii=19713. Available online: http:// www.eurosurveillance.org/ViewArticle.aspx?Articleld=19713

33. Wilson AP, Smyth D, Moore G, Singleton J, Jackson R, Gant V, et al. The impact of enhanced cleaning within the intensive care unit on contamination of the near-patient environment with hospital pathogens: a randomized crossover study in critical care untis in two hospitals. Crit Care Med. 2011;39(4):651-8.

34. Vaux S, Carbonne A, Thiolet JM, Jarlier V, Coignard B, RAISIN and Expert Laboratories Groups. Emergence of carbapenamseproducing Enterobacteriaceae in France, 2004 to 2011. EuroSurveill. 2011;16(22):pii=19880. Available from: http:// www.eurosurveillance.org/ViewArticle.aspx?Articleld=19880

35. Carbonne A, Thiolet JM, Fournier S, Fortineau N, KassisChikhani N, Boytchev I, et al. Control of a multi-hospital outbreak of KPC-producing Klebsiella pneumoniae type 2 in France, September to October 2009. Euro Surveill. 2010;15(48):pii=19734. Available from: http://www. eurosurveillance.org/ViewArticle.aspx?Articleld=19734 